\title{
EXISTENCE OF TWO SYMMETRIC SOLUTIONS FOR NEUMANN PROBLEMS
}

\author{
GHASEM A. AFROUZI, MAHNAZ BAGHERI, AND ARMIN HADJIAN
}

Received 06 April, 2016

\begin{abstract}
In this paper, we investigate the existence of at least two distinct cylindrically symmetric weak solutions for some elliptic problems involving a $p$-Laplace operator, subject to Neumann boundary conditions in a strip-like domain of the Euclidean space.
\end{abstract}

2010 Mathematics Subject Classification: 35J35; 35J60

Keywords: p-Laplace operator, variational methods, critical point

\section{INTRODUCTION}

Let $\mathcal{O} \subset \mathbb{R}^{m}$ be a bounded domain with smooth boundary and $\Omega:=\mathcal{O} \times \mathbb{R}^{n}$ be a strip-like domain. Define the space of cylindrically symmetric functions by

$$
W_{c}^{1, p}(\Omega):=\left\{u \in W^{1, p}(\Omega): u(x, \cdot) \text { is radially symmetric for all } x \in \mathcal{O}\right\} .
$$

In this space, Molica Bisci and Rădulescu in [7, Theorem 2.1] studied the existence of at least three cylindrically symmetric solutions for the following elliptic Neumann problem

$$
\begin{cases}-\Delta_{p} u+|u|^{p-2} u=\lambda \alpha(x, y) f(u) & \text { in } \Omega, \\ \frac{\partial u}{\partial v}=0, & \text { on } \partial \Omega,\end{cases}
$$

where $v$ denotes the outward unit normal to $\partial \Omega, p>m+n$ is a real number, $\lambda$ is a positive real parameter and $\Delta_{p} u:=\operatorname{div}\left(|\nabla u|^{p-2} \nabla u\right)$. Moreover, $\alpha \in L^{1}(\Omega)$ is a non-negative cylindrically symmetric function and $f: \mathbb{R} \rightarrow \mathbb{R}$ is a continuous function.

In this paper, our goal is to obtain the existence of at least two distinct cylindrically symmetric weak solutions for problem (1.1) under suitable conditions on $\alpha$ and $f$.

We denote by $c_{p}$ the best embedding constant of $W_{c}^{1, p}(\Omega)$ into $L^{\infty}(\Omega)$, i.e.,

$$
c_{p}:=\sup _{u \in W^{1, p}(\Omega)} \frac{\|u\|_{L^{\infty}(\Omega)}}{\|u\|_{W^{1, p}(\Omega)}},
$$


where

$$
\|u\|_{L^{\infty}}:=\operatorname{esssup}_{(x, y) \in \Omega}|u(x, y)|
$$

see [4, Theorem 2.2]. Further, Let $\alpha \in L^{1}(\Omega)$ is a non-negative cylindrically symmetric function such that

$$
\alpha_{0}:=\inf _{(x, y) \in \Omega} \alpha(x, y)>0,
$$

and $f: \mathbb{R} \rightarrow \mathbb{R}$ be a continuous function satisfying the following condition:

$$
|f(t)| \leq a_{1}+a_{2}|t|^{s-1}, \quad \forall t \in \mathbb{R},
$$

for some non-negative constants $a_{1}, a_{2}$ and $s>p$. We put $F(\xi):=\int_{0}^{\xi} f(t) d t$, for every $\xi \in \mathbb{R}$. Moreover, we introduce the functional $I_{\lambda}: W^{1, p}(\Omega) \rightarrow \mathbb{R}$ associated with problem (1.1),

$$
\begin{gathered}
I_{\lambda}(u):= \\
\frac{1}{p}\left(\int_{\Omega}|\nabla u(x, y)|^{p} d x d y+\int_{\Omega}|u(x, y)|^{p} d x d y\right)-\lambda \int_{\Omega} \alpha(x, y) F(u(x, y)) d x d y .
\end{gathered}
$$

Fixing the real parameter $\lambda$, a function $u \in W^{1, p}(\Omega)$ is said to be a weak solution of (1.1) if for all $v \in W^{1, p}(\Omega)$,

$$
\begin{aligned}
& \int_{\Omega}|\nabla u(x, y)|^{p-2} \nabla u(x, y) \cdot \nabla v(x, y) d x d y+\int_{\Omega}|u(x, y)|^{p-2} u(x, y) v(x, y) d x d y \\
& =\lambda \int_{\Omega} \alpha(x, y) f(u(x, y)) v(x, y) d x d y .
\end{aligned}
$$

Hence, the critical points of $I_{\lambda}$ are exactly the weak solutions of problem (1.1).

Definition 1. A Gâteaux differentiable function $I$ satisfies the Palais-Smale condition (in short (PS)-condition) if any sequence $\left\{u_{n}\right\}$ such that

(a) $\left\{I_{\lambda}\left(u_{n}\right)\right\}$ is bounded,

(b) $\left\|I_{\lambda}^{\prime}\left(u_{n}\right)\right\|_{X^{*}} \rightarrow 0, \quad$ as $n \rightarrow \infty$,

has a convergent subsequence.

We shall prove our results applying the following critical point theorem, which is a more precise version of Ricceri's variational principle [12, Theorem 2.5]. We point out that Ricceri's variational principle generalizes the celebrated three critical point theorem of Pucci and Serrin $[9,10]$ and is an useful result that gives alternatives for the multiplicity of critical points of certain functions depending on a parameter.

Theorem 1 (see [2, Theorem 3.2]). Let $X$ be a real Banach space and let $\Phi, \Psi$ : $X \rightarrow \mathbb{R}$ be two continuously Gâteaux differentiable functionals such that $\Phi$ is bounded from below and $\Phi(0)=\Psi(0)=0$. Fix $r>0$ such that $\sup _{u \in \Phi^{-1}(]-\infty, r[)} \Psi(u)<+\infty$ and assume that, for each

$$
\lambda \in] 0, \frac{r}{\sup _{u \in \Phi^{-1}(]-\infty, r D} \Psi(u)}[,
$$


the functional $I_{\lambda}:=\Phi-\lambda \Psi$ satisfies (PS)-condition and it is unbounded from below. Then, for each $\lambda \in] 0, \frac{r}{\sup _{u \in \Phi^{-1}(]-\infty, r[)} \Psi(u)}\left[\right.$, the functional $I_{\lambda}$ admits two distinct critical points.

For completeness, we refer the interested reader to the recent papers $[3,6]$ where Ricceri's variational principle has been developed on studying nonlinear Neumann problems. See also [1,5].

\section{MAIN RESULTS}

In this section we establish the main abstract result of this paper. We recall that $c_{p}$ is the constant of the continuous embedding $W_{c}^{1, p}(\Omega) \hookrightarrow L^{\infty}(\Omega)$; see (1.2).

Theorem 2. Let $f: \mathbb{R} \rightarrow \mathbb{R}$ be a continuous function satisfying condition $\left(\mathrm{f}_{1}\right)$. Moreover, assume that

( $\left.\mathrm{f}_{2}\right)$ there exist two constants $\eta>p$ and $L>0$ such that

$$
0<\eta F(t) \leq t f(t), \quad|t| \geq L .
$$

Then, for each $\lambda \in] 0, \lambda^{\star}$, problem (1.1) admits at least two distinct cylindrically symmetric weak solutions, where

$$
\lambda^{\star}:=\frac{s}{\left(s a_{1} c_{p} p^{1 / p}+a_{2} c_{p}^{s} p^{s / p}\right)\|\alpha\|_{L^{1}}} .
$$

Proof. Our aim is to apply Theorem 1 to problem (1.1) in the case $r=1$ to the Banach space $X:=W_{c}^{1, p}(\Omega)$ endowed with the norm

$$
\|u\|_{W^{1, p}}:=\left(\int_{\Omega}|\nabla u(x, y)|^{p} d x d y+\int_{\Omega}|u(x, y)|^{p} d x d y\right)^{1 / p} .
$$

For every $u \in X$ we set

$$
\Phi(u):=\frac{\|u\|_{W^{1, p}}^{p}}{p}, \quad \Psi(u):=\int_{\Omega} \alpha(x, y) F(u(x, y)) d x d y .
$$

Clearly $\Phi$ and $\Psi$ are continuously Gâteaux differentiable and

$$
\begin{gathered}
\Phi^{\prime}(u)(v):= \\
\int_{\Omega}|\nabla u(x, y)|^{p-2} \nabla u(x, y) \cdot \nabla v(x, y) d x d y+\int_{\Omega}|u(x, y)|^{p-2} u(x, y) v(x, y) d x d y,
\end{gathered}
$$

and

$$
\Psi^{\prime}(u)(v):=\int_{\Omega} \alpha(x, y) f(u(x, y)) v(x, y) d x d y,
$$

for every $v \in X$. Moreover, $\Phi^{\prime}$ admits a continuous inverse on $X^{*}$ and $\Psi^{\prime}$ is a compact operator. 
Now we prove that $I_{\lambda}:=\Phi-\lambda \Psi$ satisfies (PS)-condition for every $\lambda>0$. Namely, we will prove that any sequence $\left\{u_{n}\right\} \subset X$ satisfying

$$
m:=\sup _{n} I_{\lambda}\left(u_{n}\right)<+\infty, \quad \lim _{n \rightarrow+\infty}\left\|I_{\lambda}^{\prime}\left(u_{n}\right)\right\|_{X^{*}}=0,
$$

contains a convergent subsequence. From above, we can actually assume that

$$
\left|\frac{1}{\eta}\left\langle I_{\lambda}^{\prime}\left(u_{n}\right), u_{n}\right\rangle\right| \leq\left\|u_{n}\right\|_{W_{1, p}} .
$$

For $n$ large enough, we have

$$
\begin{aligned}
m \geq I_{\lambda}\left(u_{n}\right) & =\frac{1}{p}\left(\int_{\Omega}\left|\nabla u_{n}(x, y)\right|^{p} d x d y+\int_{\Omega}\left|u_{n}(x, y)\right|^{p} d x d y\right) \\
& -\lambda \int_{\Omega} \alpha(x, y) F\left(u_{n}(x, y)\right) d x d y
\end{aligned}
$$

then

$$
\begin{aligned}
I_{\lambda}\left(u_{n}\right) & \geq \frac{1}{p}\left(\int_{\Omega}\left|\nabla u_{n}(x, y)\right|^{p} d x d y+\int_{\Omega}\left|u_{n}(x, y)\right|^{p} d x d y\right) \\
& -\frac{\lambda}{\eta} \int_{\Omega} \alpha(x, y) f\left(u_{n}(x, y)\right) u_{n}(x, y) d x d y \\
& =\left(\frac{1}{p}-\frac{1}{\eta}\right)\left(\int_{\Omega}\left|\nabla u_{n}(x, y)\right|^{p} d x d y+\int_{\Omega}\left|u_{n}(x, y)\right|^{p} d x d y\right) \\
& +\frac{1}{\eta}\left(\int_{\Omega}\left|\nabla u_{n}(x, y)\right|^{p} d x d y+\int_{\Omega}\left|u_{n}(x, y)\right|^{p} d x d y\right. \\
& \left.-\lambda \int_{\Omega} \alpha(x, y) f\left(u_{n}(x, y)\right) u_{n}(x, y) d x d y\right) \\
& =\left(\frac{1}{p}-\frac{1}{\eta}\right)\left\|u_{n}\right\|_{W^{1, p}}^{p}+\frac{1}{\eta}\left\langle I_{\lambda}^{\prime}\left(u_{n}\right), u_{n}\right\rangle .
\end{aligned}
$$

Thus,

$$
m+\left\|u_{n}\right\|_{W^{1, p}} \geq I_{\lambda}\left(u_{n}\right)-\frac{1}{\eta}\left\langle I_{\lambda}^{\prime}\left(u_{n}\right), u_{n}\right\rangle \geq\left(\frac{1}{p}-\frac{1}{\eta}\right)\left\|u_{n}\right\|_{W^{1, p}}^{p} .
$$

Consequently, $\left\{\left\|u_{n}\right\|\right\}$ is bounded. By the Eberlian-Smulyan theorem, without loss of generality, we assume that $u_{n} \rightarrow u$. Then $\Psi^{\prime}\left(u_{n}\right) \rightarrow \Psi^{\prime}(u)$ because of compactness. Since $I_{\lambda}^{\prime}\left(u_{n}\right)=\Phi^{\prime}\left(u_{n}\right)-\lambda \Psi^{\prime}\left(u_{n}\right) \rightarrow 0$, then $\Phi^{\prime}\left(u_{n}\right) \rightarrow \lambda \Psi^{\prime}(u)$. Since $\Phi^{\prime}$ has a continuous inverse, then $u_{n} \rightarrow u$ and so $I_{\lambda}$ satisfies (PS)-condition.

From $\left(\mathrm{f}_{2}\right)$, there is a positive constant $C$ such that

$$
F(t) \geq C|t|^{\eta}
$$

for all $|t|>L$. In fact, setting $b:=\min _{|\xi|=L} F(\xi)$ and

$$
\varphi_{t}(\beta):=F(\beta t), \quad \forall \beta>0,
$$


by $\left(f_{2}\right)$, for every $|t|>L$ one has

$$
0<\eta \varphi_{t}(\beta)=\eta F(\beta t) \leq \beta t \cdot f(\beta t)=\beta \varphi_{t}^{\prime}(\beta), \quad \forall \beta>\frac{L}{|t|} .
$$

Therefore,

$$
\int_{L /|t|}^{1} \frac{\varphi_{t}^{\prime}(\beta)}{\varphi_{t}(\beta)} d \beta \geq \int_{L /|t|}^{1} \frac{\eta}{\beta} d \beta
$$

Then

$$
\varphi_{t}(1) \geq \varphi_{t}\left(\frac{L}{|t|}\right) \frac{|t|^{\eta}}{L^{\eta}} .
$$

Taking into account of (2.2), we obtain

$$
F(t) \geq F\left(\frac{L}{|t|} t\right) \frac{|t|^{\eta}}{L^{\eta}} \geq b \frac{|t|^{\eta}}{L^{\eta}} \geq C|t|^{\eta},
$$

where $C>0$ is a constant. Thus, (2.1) is proved.

Fixed $u_{0} \in X \backslash\{0\}$, for each $t>1$ one has

$$
I_{\lambda}\left(t u_{0}\right) \leq \frac{1}{p} t^{p}\left\|u_{0}\right\|_{W^{1, p}}^{p}-\lambda \alpha_{0} C t^{\eta} \int_{\Omega}\left|u_{0}(x, y)\right|^{\eta} d x d y .
$$

Since $\eta>p$, this condition guarantees that $I_{\lambda}$ is unbounded from below. Fixed $\lambda \in$ ] $0, \lambda^{\star}[$, from definition of $\Phi$ it follows that

$$
\|u\|_{W^{1, p}}<p^{1 / p},
$$

for each $u \in X$ such that $u \in \Phi^{-1}(]-\infty, 1\left[\right.$ ). Moreover, ( $\left.\mathrm{f}_{1}\right)$, the compact embedding $X \hookrightarrow L^{\infty}(\Omega)$ and (2.3) imply that, for each $u \in \Phi^{-1}(]-\infty, 1[)$, we have

$$
\begin{aligned}
\Psi(u) & \leq \int_{\Omega} \alpha(x, y)\left(a_{1}|u(x, y)|+\frac{a_{2}}{s}|u(x, y)|^{s}\right) d x d y \\
& \leq\left(a_{1}\|u\|_{L^{\infty}}+\frac{a_{2}}{s}\|u\|_{L^{\infty}}^{s}\right)\|\alpha\|_{L^{1}} \\
& \leq\left(a_{1} c_{p}\|u\|_{W^{1, p}}+\frac{a_{2} c_{p}^{s}}{s}\|u\|_{W^{1, p}}^{s}\right)\|\alpha\|_{L^{1}} \\
& <\left(a_{1} c_{p} p^{1 / p}+\frac{a_{2}}{s} c_{p}^{s} p^{s / p}\right)\|\alpha\|_{L^{1}},
\end{aligned}
$$

and so,

$$
\sup _{u \in \Phi^{-1}(]-\infty, 1[)} \Psi(u) \leq\left(a_{1} c_{p} p^{1 / p}+\frac{a_{2}}{s} c_{p}^{s} p^{s / p}\right)\|\alpha\|_{L^{1}}=\frac{1}{\lambda^{*}}<\frac{1}{\lambda}
$$

From (2.4) one has

$$
\lambda \in] 0, \lambda^{\star}[\subseteq] 0, \frac{1}{\sup _{u \in \Phi^{-1}(]-\infty, 1[)} \Psi(u)}[.
$$


Hence, Theorem 1.2 assures the existence of at least two distinct critical points for problem (1.1). Also, it is proved in [7, proof of Theorem 2.1] that $I_{\lambda}$ is an invariant functional with respect to the action of the compact group of linear isometries of $\mathbb{R}^{n}$. Thus, we can apply the principle of symmetric criticality (see [8]) to the smooth and isometric invariant functional $I_{\lambda}$ and deduce that problem (1.1) admits at least two distinct cylindrically symmetric weak solutions. The proof is complete.

Remark 1. We observe that, if $f$ is non-negative and $f(0) \neq 0$, then Theorem 2 ensures the existence of two positive cylindrically symmetric weak solutions for problem (1.1) (see, e.g., [11, Theorem 11.1]).

\section{REFERENCES}

[1] L. Barbu and C. Enache, "Maximum principles, Liouville-type theorems and symmetry results for a general class of quasilinear anisotropic equations." Adv. Nonlinear Anal., vol. 5, no. 4, pp. 395-405, 2016, doi: 10.1515/anona-2015-0127.

[2] G. Bonanno, "Relations between the mountain pass theorem and local minima." Adv. Nonlinear Anal., vol. 1, no. 3, pp. 205-220, 2012, doi: 10.1515/anona-2012-0003.

[3] G. Bonanno, G. Molica-Bisci, and V. Rădulescu, "Weak solutions and energy estimates for a class of nonlinear elliptic Neumann problems.” Adv. Nonlinear Stud., vol. 13, no. 2, pp. 373-389, 2013, doi: 10.1515/ans-2013-0207.

[4] F. Faraci, A. Iannizzotto, and A. Kristály, "Low-dimensional compact embeddings of symmetric Sobolev spaces with applications.” Proc. Roy. Soc. Edinburgh Sect. A, vol. 141, no. 2, pp. 383 395, 2011, doi: 10.1017/S0308210510000168.

[5] N. Labropoulos and V. Rădulescu, "On the best constants in Sobolev inequalities on the solid torus in the limit case $p=1 . "$ Adv. Nonlinear Anal., vol. 5, no. 3, pp. 261-291, 2016, doi: 10.1515/anona-2015-0125.

[6] G. Molica-Bisci and D. Repovš, "Nonlinear Neumann problems driven by a nonhomogeneous differential operator." Bull. Math. Soc. Sci. Math. Roumanie (N.S.), vol. 57(105), no. 1, pp. 13-25, 2014.

[7] G. Molica-Bisci and V. Rădulescu, "Multiple symmetric solutions for a Neumann problem with lack of compactness." C. R. Math. Acad. Sci. Paris, vol. 351, no. 1-2, pp. 37-42, 2013, doi: 10.1016/j.crma.2012.12.001.

[8] R. Palais, "The principle of symmetric criticality." Comm. Math. Phys., vol. 69, no. 1, pp. 19-30, 1979, doi: 10.1007/BF01941322.

[9] P. Pucci and J. Serrin, "Extensions of the mountain pass theorem." J. Funct. Anal., vol. 59, no. 2, pp. 185-210, 1984, doi: 10.1016/0022-1236(84)90072-7.

[10] P. Pucci and J. Serrin, "A mountain pass theorem.” J. Differential Equations, vol. 60, no. 1, pp. 142-149, 1985, doi: 10.1016/0022-0396(85)90125-1.

[11] P. Pucci and J. Serrin, "The strong maximum principle revisited." J. Differential Equations, vol. 196, no. 1, pp. 1-66, 2004, doi: 10.1016/j.jde.2003.05.001.

[12] B. Ricceri, "A general variational principle and some of its applications." J. Comput. Appl. Math., vol. 113, no. 1-2, pp. 401-410, 2000, doi: 10.1016/S0377-0427(99)00269-1. 
Authors' addresses

\section{Ghasem A. Afrouzi}

Department of Mathematics, Faculty of Mathematical Sciences, University of Mazandaran, Babolsar, Iran

E-mail address: afrouzi@umz.ac.ir

Mahnaz Bagheri

Department of Mathematics, Faculty of Mathematical Sciences, University of Mazandaran, Babolsar, Iran

E-mail address: m.bagheri@yahoo.com

Armin Hadjian

Department of Mathematics, Faculty of Basic Sciences, University of Bojnord, P.O. Box 1339, Bojnord 94531, Iran

E-mail address: hadjian83@gmail.com, a.hadjian@ub.ac.ir 